\title{
VIDA Y WILDERNESS: ACTUALIDAD DE LA ÉTICA MEDIOAMBIENTAL THOREAUVIANA
}

\author{
Diego Clares \\ Universidad de Murcia \\ bttp://dx.doi.org/10.15304/ag.37.2.4343
}

\section{Resumen}

El objetivo de este artículo es defender que la obra del filósofo norteamericano Henry Thoreau es importante actualmente para la reflexión ética sobre el medioambiente. En sus obras considera con frecuencia problemas que afectan a nuestro entorno natural, así como a toda la vida en general. La importancia de su pensamiento reside en su biocentrismo y en cómo a partir de él establece unos criterios éticos. Lejos de profundizar en la propuesta thoreauviana, este artículo pretende exponer sus principales cuestiones, desarrolladas alrededor de los conceptos de 'vida' y 'wilderness', para a continuación exponer su relevancia contemporánea a través de las interpretaciones opuestas de Philip Cafaro y Daniel Botkin, y de una propuesta actual: la ecología profunda.

Palabras clave: Thoreau, ética, medioambiente, vida, wilderness, naturaleza, salvaje.

\begin{abstract}
This paper's aim is to defend that the work of North American philosopher Henry Thoreau is nowadays an influence for ethical reflection about environment. He considers frequently in his work problems that affect our natural surroundings, so all life generally. The importance of his thought lies in his biocentrism and in who founds some ethical criteria from it. Far from going deep in the thoreauvian proposal, this article intends to expose his main issues, developing them around concepts of 'life' and 'wilderness', for explaining its relevance through two contrary understandings, by Philip Cafaro and Daniel Botkin, and a current proposal: deep ecology.

Keywords: Thoreau, ethics, environment, life, wilderness, nature, wild.
\end{abstract}

Recibido: 04/10/2017. Aceptado: 20/12/2017. 


\section{Introducción}

La ética medioambiental (o ética del trato con el entorno natural) ha sufrido una gran expansión en el último siglo; pero ya antes, como señala Phillip Cafaro, había importantes reflexiones sobre sus principales cuestiones. Uno de los primeros en plantearlos fue el filósofo Henry David Thoreau, quien vivió hace dos siglos en Concord, Massachusetts.

Thoreau se caracteriza por ser un autor asistemático, alejado de la filosofía académica y de los grandes sistemas occidentales. Sus ensayos suelen dividirse en dos temáticas: natural y social, pero muy a menudo encontramos que dentro de estos dos grupos hay numerosas cuestiones éticas, políticas o epistémicas, entre otras. Sus escritos siguen un esquema propio, adaptado a cada tema y desarrollado deliberadamente, aunque casi nunca sean explícitos. Por eso su ética medioambiental, pese a aparecer expuesta en numerosas composiciones, no se configura como un único sistema, sino como varios desarrollos parciales. Entre éstos, atenderemos a tres: la reseña "Paraíso (para ser) recuperado" ("Paradise (to be) Regained", 1843), que es una de las primeras reflexiones que realiza sobre el tema; su obra más importante, Walden (1854); y el ensayo "Pasear" ("Walking", 1862). Prestaremos atención, al comentar este último ensayo, a la noción de 'wildness', muy vinculada a su concepción de la vida, y a la de 'wilderness', bajo la premisa de que constituyen, en un sentido estricto, la propuesta más originalmente thoreauviana que podemos encontrar en su ética medioambiental.

En la segunda parte de este artículo mostraremos cómo esta propuesta aún puede ser tenida en cuenta para arrojar cierta luz ante los problemas que se plantean actualmente en la ética medioambiental.

\section{Tres momentos de una ética medioambiental asistemática}

La ética medioambiental thoreauviana está compuesta por múltiples textos en los que el filósofo de Concord desarrolla diferentes cuestiones íntimamente relacionadas. Por eso no se trata de una ética sistemática, ni una ética reductible a un único sistema. El objetivo que perseguimos en este apartado es doble: primero, exponer tres importantes momentos de este desarrollo de su ética medioambiental, y segundo, hacer manifiesto que la postura de Thoreau varía y que estos tres momentos tienen incluso aspectos incompatibles que no podrían formar parte del mismo sistema ético si lo tomáramos en sentido estricto. Conviene, por lo tanto, considerar qué ele- 
mentos son los principales de cada momento, cuáles se mantienen y cuáles varían, y por qué.

\section{Vestigios de una ética transcendentalista}

Podemos decir que el primer texto en el que Thoreau plantea, con suficiente profundidad, cuestiones éticas de carácter medioambiental es la reseña "Paraíso (para ser) recuperado", que escribió como crítica al libro de Johann Adolphus Etzler: The Paradise with in the Reach of all Men, without Labor, by Powers of Nature and Machinery. An Address to all intelligent Men, de 1842. Thoreau hace una crítica sarcástica de la propuesta utópica de Etzler, exponiendo a la vez un principio básico: actuar conforme a nuestra naturaleza, en vez de cambiar toda la naturaleza para adaptarla a nuestros caprichos. "Paraíso (para ser) Recuperado" constituye el primer intento de Thoreau de oponerse al dominio sobre la naturaleza, es decir a la domesticidad (tameness), por lo que en su crítica a Etzler se pueden encontrar fácilmente puntos en común con los desarrollos posteriores de su ética medioambiental.

Según lo cita Thoreau, Etzler propone mejorar el planeta para aprovechar su energía y que nos dé beneficios sin que tengamos que hacer el menor esfuerzo, pudiendo servir para mejorarnos a nosotros mismos, lo que el de Concord llama «la elevación de la raza hasta sus máximos límites» ${ }^{1}$. Pretende aprovechar los movimientos de las olas y las mareas, la fuerza del viento y la luz del sol, construyendo islas móviles, como una especie de barcos en los que poder vivir, aumentando nuestra salud y retrasando la muerte $^{2}$. Thoreau se opone en dos momentos a esta propuesta: en primer lugar mostrando su afiliación a la reforma moral transcendentalista liderada por Emerson, y en segundo mostrando quela propuesta de Etzler es insatisfacible por recurrir al infinito.

La afiliación al programa emersoniano de la reforma moral queda explícita desde la primera crítica de Thoreau, donde aparecen "dos transcendentalismos":

Parece por estos y otros indicios, que hay un transcendentalismo en la mecánica tanto como en la ética. [...] Uno dice que se reformará a sí mismo, y entonces la naturaleza y las circunstancias serán justas. No nos obstruyamos a nosotros mismos, porque ésta es la mayor discrepancia. [...] El otro reformará la naturaleza y las

${ }^{1}$ H.D. Thoreau, "Paradise (to be) Regained", en The Writings of Henry David Thoreau, vol. IV, Boston, Houghton Mifflin \& Co., 1906, p. 281.

${ }^{2}$ Ibid., pp. 280-281, 288-290. 
circunstancias, y entonces el hombre será justo. No habléis tan vagamente, dice, de reformar el mundo, - - yo reformaré todo el globo ${ }^{3}$.

Para los transcendentalistas el mundo es naturaleza, pero lo que conocemos por 'naturaleza' no es más que un producto de la mente. Como diría el propio Emerson, «la naturaleza siempre se viste con los colores del espíritu» ${ }^{4}$, es decir, que el ámbito espiritual determina cómo aparece lo natural. Bajo esta idea los transcendentalistas defendieron, tal como señala Thoreau, una reforma moral consistente en cambiar la actitud humana para que así el mundo fuera mejor o cambiara en consecuencia. La postura opuesta, que el de Concord caricaturiza como un transcendentalismo en la mecánica, consiste en intentar cambiar directamente el mundo según convenga para satisfacer los problemas humanos.

La crítica de Thoreau a favor de la reforma moral y en contra de ese "transcendentalismo en la mecánica" consiste en una reducción al infinito. Por ello comienza suponiendo que, efectivamente, podamos realizar la utopía propuesta por Etzler, que según señala en su libro podría alcanzarse en diez años ${ }^{5}$. Aceptando su premisa, Thoreau proclama que no debemos sucumbir a la naturaleza: debemos controlar el clima, los malos olores, los terremotos, los gases nocivos y los volcanes ${ }^{6}$, dominar las fuerzas de la naturaleza para extraer beneficio de ellas y evitar que nos afecten negativamente, implantando una paz artificiosa; pero el de Concord quiere explorar sus consecuencias, siguiendo una idea principal en el planteamiento de Etzler: la capacidad humana para comprender, controlar y mejorar la naturaleza, y por ello afirma a continuación: «Lavaremos el agua, y calentaremos el fuego, y enfriaremos el hielo, y sostendremos la tierra. Enseñaremos a los pájaros a volar, a los peces a nadar, y a los rumiantes a mascar la comida ${ }^{7}$. Tal es la convicción de que la humanidad, por medio de su racionalidad, tiene la suficiente capacidad para controlar los procesos naturales, que no parecería descabellado incluso suponer que llegado el momento sabríamos mejorar todas estas cosas y hacer la naturaleza más eficiente. Por eso Thoreau se pregunta si no debería actuar la humanidad como una divinidad, como una entidad magnánima, protectora y sanadora de todos los males, a lo que responde con otra cuestión: «¿Qué parte de magnanimidad hay para la ballena y el castor?» ${ }^{8}$.

\footnotetext{
${ }^{3}$ Ibid., pp. 281-282.

${ }^{4}$ R. W. Emerson, Nature, Boston, Thurston, Torry \& Co., 1849, p. 9.

${ }^{5}$ H.D. Thoreau, op. cit., p. 280.

${ }^{6}$ Ibid., p. 283.

${ }^{7}$ Ibid., p. 283.

${ }^{8}$ Ibid., p. 283.
} 
Éste es el paso que da Thoreau más allá de Etzler y de la respuesta estrictamente transcendentalista, que da forma a la propuesta thoreauviana: debemos valorar la autoridad que tiene el resto de vivientes respecto a su naturaleza y a sí mismos. Este juicio supera la simple reforma moral, o al menos la lleva hacia una nueva cuestión: considerar éticamente a los individuos no humanos, en vez de dar por hecho que la razón nos autoriza para tratarlos a nuestro gusto. Uno de los precedentes más importantes para comprender la ética medioambiental thoreauviana se encuentra en este cuestionamiento de nuestra supuesta autoridad para apropiarnos de la vida de otro individuo, así como la defensa de que el resto de vivientes (e incluso de individuos inertes) tiene la misma soberanía natural que los humanos.

Desde la perspectiva thoreauviana, la propuesta de Etzler ignora que el mundo no está hecho para que lo dominemos, sino para convivir en él; convivir en el mundo implica hacer un esfuerzo, ganarse el sustento y el cobijo, y situarse al mismo nivel que la ardilla, el manzano o la laguna. Buscar sólo la comodidad significa para Thoreau no comprender en qué consiste la vida y corromperla: «El principal fallo de este libro es que solamente trata de asegurar el mayor grado de grosera comodidad y placer» ${ }^{9}$.

Tal es el Paraíso para ser Recuperado [...] El hombre nunca más se ganaría la vida con el sudor de su frente. Toda labor se reduciría a "un pequeño giro de alguna palanca”, y "sacar los artículos terminados”. Pero hay una palanca, _ioh, qué difícil de girar! ¿No podría haber una palanca sobre una palanca, - una palanca infinitamente pequeña? —estaríamos dispuestos a investigarlo ${ }^{10}$.

No importa la cantidad de trabajo que tengamos que hacer, sino el hábito y la disposición para hacerlo, pues si siempre buscamos el mínimo esfuerzo a la larga nos parecerá demasiado. Tal exigencia de comodidad por encima de la acción justa o adecuada supone, como señala Thoreau, una recurrencia al infinito irresoluble.

En vez de actuar con tal afán de comodidad, deberíamos girar nuestra manivela interior, "el primer motor en toda maquinaria, completamente indispensable para todo trabajo» ${ }^{11}$. Este motor no es otro que el moral, y dependiendo de la fuerza que tenga nos dispondrá a trabajar con más o menos ánimo. Es más conveniente trabajar con el ánimo adecuado que buscar una comodidad absoluta. La propuesta de Thoreau pasa por dar importancia a la iniciativa moral individual, pero siempre buscando un desarrollo natural

\footnotetext{
${ }^{9}$ Ibid., p. 302.

${ }^{10}$ Ibid., p. 297.

${ }^{11}$ Ibid., p. 297.
} 
y vital de carácter biocéntrico ${ }^{12}$. La reforma que defiende Thoreau se presenta como una reforma ética que predica un desarrollo personal basado en «hacer un paraíso interno que prescinda de un paraíso externo» ${ }^{13}$.

\section{Un experimento en Walden: lo indispensable para vivir}

Los dos años y dos meses que pasó Thoreau en una cabaña junto a la laguna Walden suponen la mayor parte de su actual fama. Sin embargo esta estancia fue un experimento, tal como señala Antonio Casado, para quien la cabaña es «un lugar de tránsito» ${ }^{14}$. Thoreau se propuso una actividad cuyo objetivo no era crear hábito o impartir doctrina per se, sino realizar un ejercicio crítico y terapéutico, aunque después tuviera que derribar todo lo que había construido. La "Conclusión" de Walden incide justamente sobre este carácter temporal, al afirmar: «Tenía otras muchas vidas que vivir» ${ }^{15}$.

El filósofo de Walden realiza una exposición original de la ética, planteada en ciertos sentidos desde el ambiente en vez del individuo; pretende hacer algo similar en su primer libro, Una semana en los ríos Concord y Merrimack (A Week on the Concord and Merrimack Rivers, 1849), como explican Phillip Cafaro ${ }^{16}$ y Joan Burbick ${ }^{17}$, exponiendo la historia y la experiencia desde el entorno natural y no desde lo estrictamente humano. Pero es en Walden donde encontramos por primera vez explícita tal consideración, que se desarrolla en el plano ético. En este extenso ensayo nos describe continuamente el bosque y la laguna, ya sea histórica, mitológica, social, geográfica o biológicamente. Pero no se limita a dar una descripción: Thoreau, como habitante de la wilderness, nos habla éticamente de ella, reflexiona sobre cómo dirigir nuestras acciones, sobre el papel vital de la naturaleza y los valores morales que nos transmite. Busca en el entorno natural un modo de explicar la ética que no tenga como criterio el interés humano y que enmarque nuestras acciones en el conocimiento de la natu-

${ }^{12}$ Con 'biocéntrico' me refiero a que Thoreau pone como aspecto central de su ética la vida tanto humana como del resto de seres vivos, e incluso de los inertes en cuanto que al existir tienen cierta naturaleza propia y viven formando parte de un ecosistema.

${ }^{13}$ Ibid., p. 304.

${ }^{14}$ A. Casado da Rocha, Una casa en Walden, Logroño, Pepitas ed., 2017, p. 37.

${ }^{15}$ H.D. Thoreau, "Walden”, en The Writings of Henry David Thoreau, vol. II, Boston, Houghton Mifflin \& Co., 1906, p. 355.

${ }^{16}$ P. Cafaro, Thoreau's Living Ethics, Athens, University of Georgia Press, 2004, pp. 140-141.

${ }^{17} \mathrm{~J}$. Burbick, Thoreau's Alternative History: Changing Perspectives on Nature, Culture and Language, University of Pennsylvania Press, Philadelphia 1987, pp. 15-34. 
raleza no-humana que nos rodea, de la que hemos surgido y de la que nos nutrimos.

El filósofo de Walden se sitúa como un espectador, como la primera persona que contempla y reflexiona sobre el entorno, alguien que actúa en base a la naturaleza, interviniendo por su propio sustento en la medida de lo estrictamente vital. La filosofía de Thoreau es una reflexión ética sobre la vida, y su mayor cuestión está, justamente, en la pregunta que plantea en Walden: ¿en qué consiste vivir? ${ }^{18}$ Esta pregunta no parece tener una respuesta concisa: a veces Thoreau da pautas de lo que considera requisitos mínimos para vivir, otras veces nos habla de sus hábitos diarios, pero la respuesta en conjunto de Walden no es individual, no describe lo que hace uno para vivir, sino que se trata de una respuesta sobre el entorno, sobre la naturaleza y, tal como proclamaba en "Paraíso (para ser) recuperado", reformarnos moralmente para actuar en consecuencia. Se podría concluir que en este ensayo el filósofo de Concord desarrolla, en todas las facetas que es capaz de mostrar mediante su experiencia, su concepción de la vida.

\section{a. La reforma moral: nuevas perspectivas y reflexión}

Vivir en el mundo, para los humanos, es una actividad principalmente ética que no supone sólo seguir ciertos principios, sino también educarnos en ellos para adquirirlos en la práctica. Por eso la reforma moral es, primero, una reforma educativa. Para Thoreau tal educación debe realizarse por medio de la experiencia directa y de la escritura como medio para la reflexión. Por esto intentó sacar la escritura de la biblioteca y ponerla a disposición de la wilderness, y como resultado su ética tiene una consecuencia muy cercana al ecologismo: aprender del entorno natural implica respetarlo. Si no tenemos una educación adecuada en lo que respecta a la naturaleza, el desconocimiento nos puede conducir a un comportamiento injusto. Lo que para el de Concord es una educación natural, en Walden se explica con actividades como la caza y la pesca que dejan paso, llegado el momento, a otro tipo de relaciones con el mundo. Una persona formada en el bosque, tratando con los animales salvajes, aprende el valor de la muerte y las implicaciones de arrebatar la vida o causar sufrimiento ${ }^{19}$.

La reforma moral va encaminada a conocer y respetar (o tratar en consecuencia) el mundo que habitamos, pero reconocer qué mundo habitamos es ya un paso en este sentido. En el capítulo "Soledad", el filósofo de la

\footnotetext{
${ }^{18}$ H.D. Thoreau, op. cit., pp. 100-101.

${ }^{19}$ Ibid., p. 235.
} 
laguna reclama que no podemos sentirnos ajenos a la naturaleza, no somos diferentes al resto de habitantes del mundo, aunque creamos que nuestras sociedades nos aíslan o nos dan un estatus superior al resto, y en cualquier caso sólo impiden que conozcamos nuestra naturaleza. En resumen, no se puede saber qué es vivir si no salimos de la ciudad, si nuestra perspectiva está limitada por sus muros. En una contraposición llamativa entre la naturaleza y la cultura, Thoreau revela la irrealidad de la soledad extrema ideal que podría deducirse de su experimento. De hecho, jamás llegó a dejar la sociedad: sólo la observo desde los márgenes, desde los límites entre la ciudad y la wilderness.

La observación desde el límite le permite, en "Soledad", jugar tanto con la experiencia de lo estrictamente natural como con la observación de los rastros humanos en ella. Si bien el capítulo comienza con una descripción del bosque y de sí mismo como parte de él, en el segundo párrafo cuenta que, al llegar a su cabaña, encontraba señales del paso de algún visitante: una nota en su mesa, unas ramitas peladas frente a su puerta, o el olor de una pipa $^{20}$. Esta posición le permite contemplar la vida en general desde un ángulo en el que capta tanto lo más natural como lo propiamente civilizado, y así plantear una reflexión ética que tiene más en cuenta el impacto que las actividades humanas causan sobre el entorno natural.

La ética medioambiental tiene que darse, aunque parezca obvio, en el entorno o ambiente, conociéndolo de primera mano. En otras palabras, quienes no mantienen una relación personal con el entorno natural tampoco pueden desarrollar una adecuada ética medioambiental. De esta forma, a Thoreau no le interesa sólo conocer el mundo desde la sociedad sino también desde el conocimiento directo de la misma realidad natural. Uno de los episodios más conocidos y comentados de tal conocimiento, que narra en Walden, es el de su campo de judías. Aunque se denomina a sí mismo un pitagórico en lo que respecta a las judías ${ }^{21}$, admite que al querer conocerlas por completo también las comió (pese a que prefería venderlas o cambiarlas por arroz $)^{22}$. Su relación con las judías fue tan intensa que narra cómo las defendía de las malas hierbas con su azada como si fuera un soldado, no sin plantearse:

${ }^{20}$ Ibid., p. 144.

${ }^{21}$ Ibid., p. 179. Según Diógenes Laercio, Pitágoras, que nunca comía judías (o habas), estaba huyendo (algunas historias dicen que de los siracusanos, otras que de los crotoniatas) cuando llegó a un huerto de estas legumbres, y al detenerse para no pisarlas lo encontraron y mataron (D. Laercio, Vidas de los filósofos más ilustres, VIII, "Pitágoras” 25-26).

${ }^{22}$ H.D. Thoreau, op. cit., pp. 178-179. 
¿qué derecho tengo a expulsar a la hierba de San Juan y al resto, y deshacer su antiguo jardín de hierbas? [...] ¿quién considera el valor de la cosecha que produce la naturaleza en los campos aún más salvajes sin que el hombre los reforme?23

Porque si bien la agricultura puede ser útil tanto para alimentarnos como para aproximarnos mejor a la naturaleza, hay que tomarla con cuidado. Tal es la crítica principal de Thoreau a la agricultura:

Por avaricia y egoísmo, y un hábito denigrante, del que ninguno de nosotros está libre, de considerar el suelo como una propiedad, o máxime el medio para adquirirla, el paisaje se deforma, la agricultura se degrada con nosotros, y el granjero lleva la vida más mediocre. Sólo conoce la Naturaleza como un ladrón ${ }^{24}$.

He aquí la cuestión que conduce toda la reflexión del de Concord sobre la vida: comprender que todos vivimos naturalmente, y que no han nacido especies para servir a otras sino para convivir, para ayudarse mutuamente o para devorarse unas a otras. Tal como criticaba el extremo confort propuesto por Etzler, también nos muestra aquí la degradación de la domesticidad, de utilizar el mundo como un almacén a nuestra disposición y, en consecuencia, actuar como ladrones. Vivir, en un sentido natural, implica interactuar en el mundo como cualquier otro individuo.

El primer problema que plantea Thoreau es el derecho o la legitimidad que tenemos para poseer la tierra y aprovecharnos unidireccionalmente de los productos naturales que ayudamos a cultivar. Esa misma idea del cultivo o el cuidado de la tierra está puesta en duda a lo largo del capítulo.

Más que proteger o cuidar la tierra, Thoreau defiende aquí que nos ocupemos de nuestros propios asuntos y dejemos de lado toda pretensión de intervenir en el curso natural del mundo. La intervención debe ser tan mínima que nuestros cultivos parezcan salvajes ${ }^{25}$. Sólo conociendo de cerca la naturaleza se puede actuar de la forma adecuada, en la medida que así escapamos de nuestra limitación doméstica. De ahí que el autor de Walden se pregunte: "¿Qué debo aprender de las judías, o las judías de mí?» ${ }^{26}$ y, más adelante: «¿Por qué nos preocupa tanto que germinen nuestras judías, y no nos preocupamos en absoluto por una nueva generación de hombres? ${ }^{27}$.

\footnotetext{
${ }^{23}$ Ibid., pp. 171-172, 174.

${ }^{24}$ Ibid., p. 183.

${ }^{25}$ Ibid., p. 174.

${ }^{26}$ Ibid., p. 171.

${ }^{27}$ Ibid., p. 181.
} 


\section{b. Sencillez y sobriedad}

La sencillez (o simplicidad) es el rasgo más característico de la filosofía de Thoreau; toda su ética está articulada alrededor de este concepto. La sencillez consiste en actuar según lo estrictamente indispensable para vivir:

¡Sencillez, sencillez, sencillez! Digo que reduzcáis vuestros asuntos a dos o tres, y no cien o mil; en vez de un millón contad media docena, y guardad vuestras cuentas en la uña del pulgar. [...] En vez de tres comidas al día, si es necesario comed sólo una; en vez de cien platos, cinco; y reducid otras cosas en proporción ${ }^{28}$.

Se trata de la adecuación a lo mínimo indispensable o necesario para la vida; por eso comienza Walden proponiendo que quiere encontrar lo más básico, aquello sin lo cual no se puede subsistir, para así comprender lo que es vivir al margen de cualquier aditivo ${ }^{29}$. Según el de Concord sólo desde esa perspectiva se puede descubrir qué es aquello naturalmente vital para los humanos y qué es todo aquello añadido a la vida que perjudica tanto a su sociedad como a su entorno. La sencillez conduce a lo más básico, a lo estrictamente necesario para vivir ${ }^{30}$ y que permite reflexionar éticamente. Las consecuencias de tal perspectiva se enfocan no sólo a cada persona, sino también al entorno. Thoreau entiende que lo necesario para vivir no consiste sólo en lo que cada uno adquiere o consume, sino en que lo adquiera y consuma adecuadamente, sin agotar los recursos ni agotarse a sí mismo. La sencillez es el mejor método para ello: si nos aseguramos de vivir con lo justo en cada momento (sin por ello sacrificar nuestra subsistencia), garantizamos que no agotaremos el mundo, ya que éste sigue fluyendo y regenerándose, porque está vivo ${ }^{31}$.

La sencillez se manifiesta en gran parte de la ética expuesta en Walden como sobriedad, como una abstención de ciertos lujos, ostentosos e innecesarios. Se trata del otro lado de la sencillez: para vivir con lo justo y necesario hay que identificar lo que debemos evitar. En el capítulo "Leyes superiores" expone algunos de los hábitos domésticos y ostentosos que él mismo evitó en su búsqueda de lo más natural. Entre ellos destaca su crítica a la alimentación de origen animal; ésta, según Thoreau, es algo que debería ir desapareciendo en una sociedad civilizada y bien educada ${ }^{32}$. La opulencia no sólo daña el entorno, sino que además hace que nos obcequemos con

\footnotetext{
${ }^{28}$ Ibid., pp. 101-102.

${ }^{29}$ Ibid., pp. 12-13.

${ }^{30}$ Ibid., p. 101.

${ }^{31}$ Ibid., pp. 333, 339-341, 349.

${ }^{32}$ Ibid., p. 239.
} 
nuestras propias costumbres, sin poder ver más allá de ellas ni reflexionar adecuadamente sobre nuestra intervención en el mundo. Alguien que se alimente excesivamente tiene que preocuparse más de lo necesario, centrando la atención en preparativos laboriosos, olores incómodos, limpiezas tediosas y digestiones pesadas. Con tanta molestia, Thoreau ve un gran inconveniente:

Creo que todo hombre que alguna vez haya decidido preservar sus facultades superiores o poéticas en la mejor condición ha estado inclinado específicamente a abstenerse del alimento animal, y de cualquier alimento en exceso ${ }^{33}$.

Pero la alimentación es vital, y aunque sea prudente limitarla a lo necesario no debemos limitarnos en exceso; si hace falta, dice Thoreau, se comería una rata frita ${ }^{34}$. Lo importante no es determinar qué podemos hacer y qué no, sino mostrar unos criterios adecuados a nuestras disposiciones naturales y al entorno para decidir en cada caso cuál es la acción más apropiada, la más sencilla y vital. Esto exige, de nuevo, un conocimiento adecuado del entorno y de la naturaleza, más que de las costumbres sociales.

\section{c. El final de un experimento: una costra que se desprende continuamente}

Dejé los bosques por una razón tan buena como por la que fui. Quizás me pareció que tenía otras muchas vidas que vivir, y no podía prestarle más tiempo a ésta. Es destacable cuán fácil e insensiblemente caemos en una ruta específica y nos hacemos un camino trillado ${ }^{35}$.

La estancia en Walden de Thoreau no perduró porque no estaba destinada a perdurar, o a convertirse en un asentamiento, sino que debía ser una experiencia de la que extraer nuevas ideas, rechazar las que no funcionaran y seguir adelante en otras investigaciones, en otras vidas. Su conclusión, por tanto, en lo que respecta a la ética medioambiental, es que no podemos asentarnos en una única perspectiva sobre lo que es vivir o sobre cómo tratar el entorno: el mundo está repleto de diversos individuos, y decidir sobre nuestros actos no puede ser algo que limitemos a nosotros mismos. Nuestra reflexión ética no puede ir desligada jamás de la experiencia que tenemos del mundo. Es nuestra responsabilidad decidir éticamente en base a algo más que la sociedad humana, puesto que hay más vida además de la que conocemos socialmente.

\footnotetext{
${ }^{33}$ Ibid., p. 237.

${ }^{34}$ Ibid., p. 240.

${ }^{35}$ Ibid., p. 355.
} 
La conclusión de Thoreau incide en el cambio que se nos exige como individuos con una experiencia limitada; no somos eternos ni infalibles, sino que variamos continuamente y nunca está justificado que pensemos que hemos llegado a un conocimiento absoluto ni a un juicio universalmente válido, debemos seguir contrastándolo en la experiencia. El autor de Walden utiliza muchas expresiones con las que se refiere a esta ruptura entre la costumbre y el cambio: el camino trillado, el día que queda por amanecer y la influencia de la primavera en nuestros pensamientos ${ }^{36}$ entre otras, y, de forma muy llamativa, la costra que se desprende continuamente, con la que refleja la actitud fundamental de su ética:

John Farmer se sentó en su puerta una tarde de septiembre, después de un duro día de trabajo, aún dándole algunas vueltas mentalmente a su faena. [...] No había atendido al rumbo de sus pensamientos cuando escuchó a alguien tocando una flauta [...] Todavía pensaba en su trabajo; pero el peso de su pensamiento, aunque seguía dando vueltas en su cabeza [...] ya le interesaba muy poco. No era más que la costra de su piel, que constantemente se desprendía. [...] [sus notas] se llevaban suavemente la calle, y la ciudad, y el estado en el que vivía ${ }^{37}$.

\section{Wilderness y el arte de Pasear}

El 11 de noviembre de 1851 (casi tres años antes de publicar Walden) Thoreau anota en su diario una expresión muy similar a la citada anteriormente $^{38}$ :

Vivir mucho a la intemperie, al sol y al viento, sin duda, producirá cierta rudeza de carácter [...] Como el exceso de trabajo manual produce callos en la mano y la priva de la exquisitez del tacto. Pero creo que es una costra que se caerá suficientemente rápido, —que el remedio natural se encuentra en la proporción en que la noche sostiene al día, el inverno al verano, etc., el pensamiento a la experiencia ${ }^{39}$.

Casi íntegra aparece esta cita en "Pasear", cuyo contenido había expuesto Thoreau en numerosas conferencias. La clave del doble sentido de esta metáfora está en la idea final: el pensamiento soporta o contiene a la experiencia, como una costra que protege la piel sensible, o como la noche que permite descansar de la actividad diurna, o el invierno que conserva la vida en hibernación. Es una protección que no puede ser definitiva, puesto

${ }^{36}$ Ibid., pp. 355, 367, 346.

${ }^{37}$ Ibid., pp. 245-246. La cursiva es mía.

${ }^{38}$ Encontramos varias referencias a esta metáfora en otras entradas de su diario en 1851 y 1852.

${ }^{39}$ H.D. Thoreau, The Writings of Henry David Thoreau: Journal, vol. III, Houghton Mifflin \& Co., Boston 1906, pp. 104-105. La cursiva es mía. 
que la experiencia (como el día y como el verano) continúa y trae nueva actividad, nuevo conocimiento y nuevas ideas. La reflexión ética, como se concluye en Walden, no puede quedar estancada en unos juicios definitivos. Si bien Thoreau manifiesta claramente bastantes criterios éticos recto al medioambiente, éstos tienen que entenderse en el marco de una actividad vital continua, tan duradera como la vida misma, y no como una doctrina que pueda establecerse desde las bibliotecas. Sobre este criterio ético general va a tratar en su mayor parte "Pasear", donde el 'arte de Pasear' puede comprenderse como una virtud thoreauviana, pero sobre todo como una actividad terapéutica que nos acerca al entorno natural y nos invita a reflexionar éticamente.

\section{a. Un individuo silvestre y salvaje}

La salvajez o wildness es para Thoreau la clave del mantenimiento de la naturaleza y la vida, pues se trata de la característica principal de lo que se hace para satisfacer la propia naturaleza, para vivir en el sentido más estricto; por eso «la vida consiste en la salvajez» ${ }^{40}$. En Walden afirma: «Necesitamos el tónico de la salvajez»" ${ }^{41}$ y en "Pasear" añade que "en la Salvajez está la conservación del mundo» ${ }^{42}$.

La salvajez es un elemento dentro de lo que es éticamente bueno. Para el filósofo de Concord «todas las cosas buenas son salvajes y libres», es decir que lo éticamente bueno ha de provenir de una libre elección según lo salvaje. Ahora bien, ¿qué significa específicamente que tal acción sea salvaje? Sobre ello da esta explicación:

Hay algo en un acorde musical, ya lo produzca un instrumento o la voz humana [...], que por su salvajez, hablando sin sátira, me recuerda a los gritos [o llantos] emitidos por las bestias salvajes en sus bosques nativos. Tienen más salvajez de la que puedo comprender. [...] La salvajez del silvestre es sólo un símbolo borroso de la imponente ferocidad con la que se encuentran los hombres buenos y los amantes. ${ }^{44}$

Hay aquí varias claves: primero, la salvajez se observa o se extrae de los hábitos más instintivos como son los gritos o los llantos (o de la música en cuanto que se asemeja a éstos); en segundo lugar, la comprensión de la misma no depende simplemente de identificarla, sino que está ligada igual-

${ }^{40}$ H.D. Thoreau, "Walking”, en The Writings of Henry David Thoreau, vol. V, Boston, Houghton Mifflin \& Co., 1906, p. 226.

${ }^{41}$ H.D. Thoreau, "Walden", op. cit., p. 350.

${ }^{42}$ H.D. Thoreau, "Walking”, op. cit., p. 224.

${ }^{43}$ Ibid., p. 234.

${ }^{44}$ Ibid. 
mente a instintos y, por tanto, requiere cierta interiorización; y por último afirma que la salvajez es un simbolo de ciertas actitudes, es decir, una forma de nombrar cierto comportamiento. Aunque pueda parecer una explicación circular (ya que retorna en la definición de la salvajez a la bondad), añade la idea de que se trata de un símbolo, de una forma borrosa, tenue o imprecisa, de nombrar el comportamiento feroz, desenfrenado, de las acciones que se realizan ética y pasionalmente, desatando los instintos y dejando que se desarrollen en el mundo. Esto es para Thoreau lo que hace que se conserve el mundo: no domesticar las especies ni cultivarlas ni mantenerlas a nuestro cuidado de algún modo, sino dejar que los instintos (también de los humanos) se desaten con ferocidad; porque todos «tenemos un silvestre salvaje en nuestro interior ${ }^{45}$.

\section{b. Derechos nativos}

Uno de los puntos importantes en "Pasear" que puede ser tenido en cuenta por la ética medioambiental actual es la defensa de los 'derechos nativos' (native rights), que hay que considerar teniendo en cuenta el lenguaje retórico del filósofo, que no era ajeno a la distinción entre los derechos como normas sociales y la disposición natural para realizar ciertos actos que, por semejanza a nuestra capacidad legal, se llama a veces 'derecho'. Cuando afirma:

Incluso amo ver a los animales domésticos reafirmando sus derechos nativos, cualquier evidencia de que no han perdido por completo sus hábitos salvajes y vigor originarios $^{46}$.

El filósofo de Concord tiene en el punto de mira la duda ética de si tenemos legitimidad para hacer lo que se nos antoje con el mundo, o si por el contrario carecemos de autoridad o derecho para ello. El término 'derecho' tiene aquí un sentido natural, no legal, que refuerza la idea de que cada individuo puede actuar por sí mismo en virtud de que vive. Thoreau no está pensando en que lo natural pueda tener derechos en un sentido estrictamente civil, ya que se trata de un constructo humano; por eso, cuando habla aquí de los derechos nativos se refiere en un sentido metafórico a algo similar a lo que legalmente consideramos un derecho: una legitimación natural por existir en el mundo, o por vivir, que tiene cualquier individuo sobre sí mismo. Dicho de un modo más claro: todos los seres vivos poseen la capacidad de actuar por sí mismos (ya sea de forma meditada o siguiendo

\footnotetext{
${ }^{45}$ Ibid., p. 237.

${ }^{46}$ Ibid., p. 234.
} 
algún instinto), y al hacerlo frente a la domesticación (o la imposición de hábitos por parte de la civilización) afirman tal capacidad como una autoridad sobre sí mismos. Volviendo al contexto de esta idea, lo que reclama Thoreau es que cada individuo coexiste con los demás según su naturaleza y no tenemos justificación ética para quebrantarla o adaptarla según nuestras leyes (incluso aunque entendamos que éstas son alguna "segunda" naturaleza), porque la naturaleza no funciona de tal modo: un individuo puede, por naturaleza, limitar la acción de otro (por ejemplo cazándolo u ocupando su territorio) por un motivo vital (como alimentarse), pero esto no limita su naturaleza. Lo que hace la domesticidad, a ojos de Thoreau, es intentar corromper el instinto natural en beneficio propio, no dejando que los individuos desarrollen sus "derechos nativos". De tal modo, aunque haya especies más dóciles, dice el de Concord: "Que el resto puedan reducirse al mismo nivel no es una razón para que se deban quebrantar sus naturalezas» ${ }^{47}$.

\section{c. Aprehender ${ }^{48}$ en la wilderness}

Así como el pato salvaje es más veloz y bello que el doméstico, también lo es el pensamiento el ánade ${ }^{49}$ salvaje, que mientras cae el rocío alza su camino sobre los pantanos $^{50}$.

En "Pasear" defiende Thoreau que una ética comprometida con el ambiente no sólo tiene que valorarlo por lo que es, sino que además tiene que conocerlo, y la mejor forma de hacerlo consiste en acercarse a él paseando y observándolo con el cuidado de un naturalista, actividad que Thoreau perfeccionó con los años. Aprehender del entorno natural, de la wilderness, nos ayuda a realizar una mejor ética que lo tenga en cuenta, que respete la naturaleza y permita la salvajez. Si bien decíamos sobre la vida que legitima a los individuos a actuar siguiendo su naturaleza (i.e. salvajemente), la wilderness es el lugar donde hemos de aprehender tal naturaleza, así como de ella y de sus circunstancias. La importancia de la conservación de los espacios naturales reside, para Thoreau, en esta ética que podríamos calificar

${ }^{47}$ Ibid., p. 235.

48 Con este término nos referimos a captar o adquirir conocimiento mediante la experiencia sensible, en contraposición al aprendizaje estrictamente teórico.

${ }^{49} \mathrm{El}$ ánade, o ánade real, era considerado por los indios pawnees como un guía que conocía todos los caminos del cielo, el agua y la tierra (J. Chevalier, Diccionario de símbolos, Barcelona, Herder, 1986, p. 806). Es posible que por ello Thoreau los utilice también como guías del pensamiento.

${ }^{50}$ H.D. Thoreau, op. cit., p. 231. 
de vitalista o biocéntrica, y en que sólo mediante la conservación de la wilderness se pude conservar la diversidad natural. Pero en última instancia tal conservación debe ser un distanciamiento, un evitar la domesticidad. Evitando destruir la naturaleza de cada individuo y del mundo en general, dice Thoreau, se permite que se perpetúe por sí misma, y esto es lo más valioso. Como afirma en "Pasear", el mundo sólo se conserva mediante lo salvaje ${ }^{51}$.

\section{Actualidad y praxis}

\section{a. Biocentrismo o antropocentrismo}

Hemos de atender ahora a la relevancia de esta postura dentro de las discusiones contemporáneas. Sin embargo, debido a que las posiciones teóricas actuales sobre el medioambiente son muy posteriores a la de Thoreau, no sería correcto encuadrarlo sin más en una de ellas. Considerándolo como posible influencia, conviene revisar la cercanía y el parentesco, pues incluso sin una relación directa no cabe duda de que aún hoy el filósofo de Concord ofrece un buen punto de apoyo para este tipo de cuestiones éticas. Como primera aproximación, consideremos que Phillip Cafaro vincula a Thoreau con las propuestas de Aldo Leopold y Rachel Carson, siendo ambos importantes antecedentes del movimiento ecologista actual ${ }^{52}$.

Ahora bien, entre los intérpretes del filósofo de Concord no hay un acuerdo sobre en qué teoría se le puede encuadrar, ya que hay interpretaciones contrarias, e incluso algunas que niegan la pertinencia de indagar en esta vía. Para continuar con el propósito del presente trabajo, voy a considerar la interpretación de dos autores: Philip Cafaro y Daniel Botkin.

Cafaro señala a Thoreau, en sentido general, como un 'ético de la virtud medioambiental' (environmental virtue ethicist) ${ }^{53}$. Esta terminología es muy genérica, y no sin motivo: no es fácil ni correcto asignar una corriente actual al pensamiento thoreauviano, que se desarrolla un siglo antes de que las discusiones sobre el entorno natural adquieran importancia. Por ello sólo refiere al tema general (el medioambiente, o entorno natural) y a la forma o método que adopta (las virtudes). Cafaro no es ajeno a la variedad de propuestas que podrían denominarse, de este modo, con el calificativo

${ }^{51}$ Ibid., p. 224.

${ }^{52}$ P. Cafaro, "Thoreau, Leopold, and Carson: Toward an Environmental Virtue Ethics", Environmental Ethics, 23:1 (2001), 3-17. A. Dobson, Pensamiento politico verde, Barcelona, Paidós, 1997, pp. 59-60, 75-76.

${ }^{53}$ P. Cafaro, op. cit., p. 5. 
'environmental', pero relacionar a Thoreau con una de ellas es arriesgado. A la hora de precisar la propuesta thoreauviana, Cafaro insiste sobre todo en cuatro aspectos:

$(\alpha)$ Mantiene un interés económico, centrado en el consumo responsable ${ }^{54}$. Se refiere, en sentido clásico, a la gestión de los recursos que proporciona el entorno.

( $\beta$ ) Realiza estudios científicos consciente de cierta limitación ${ }^{55}$. Tal acercamiento científico es sobre todo personal, y que no sólo un estudio objetivo de la naturaleza.

$(\gamma)$ Se opone al antropocentrismo ${ }^{56}$, es decir, a considerar la ética desde una perspectiva exclusivamente humana, o como una deliberación estrictamente sobre lo humano.

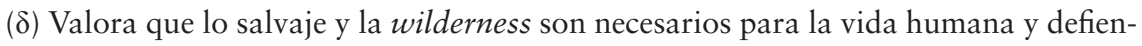
de su conservación ${ }^{57}$.

Pese a que Cafaro afirma en varias ocasiones que Thoreau se opone al antropocentrismo, en Thoreau's Living Ethics advierte que algunas de sus ideas son racionalistas, anti-sensualistas, y caen en una idolatría filosófica hacia la razón ${ }^{58}$. Pero si el de Concord hiciera tal cosa, entraría en conflicto con la crítica al antropocentrismo y con la mayoría de aspectos que hemos señalado anteriormente, y sobre todo se tambalearía la centralidad de la vida y de la experiencia de la wilderness en su ética medioambiental. Sin embargo, la justificación de que en la obra de Thoreau tenga a veces tanto protagonismo la razón sobre los sentidos proviene de una antigua influencia: desde que Aristóteles describiera a los humanos como animales racionales hay consenso en la concepción de que, al menos, la posición de la razón está privilegiada en los humanos por circunstancias particulares de nuestra naturaleza. Pero esto no implica concluir que en el mundo domina la razón sobre todo lo demás, puesto que para Thoreau hay un claro predominio de la vida en general, también en su irracionalidad: por eso su ética es biocéntrica y no racionalista, ya que de serlo dejaría fuera de consideración la vida ajena a los humanos, lo que ya hemos visto que no hace. El hecho de que la razón tenga un predominio en la vida humana no impide que, al hablar de la naturaleza en general, prevalezcan otros criterios.

Daniel Botkin, sin embargo, no coincide con esta interpretación, sino que considera que el único motivo, según Thoreau, para conservar la wil-

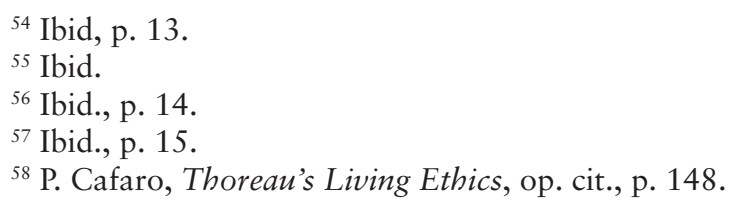


derness es nuestra propia espiritualidad. En No Man's Garden lo vincula a una tradición marcada por el dualismo platónico y cartesiano que enfatiza «la individualidad, el humanismo, la razón, la racionalidad, y la democracia las mismas características que Thoreau apreciaba ${ }^{59}$. Y más adelante insiste en que el de Concord «valoraba la naturaleza por sus cualidades espirituales, creativas, y religiosas no huía de tales intangibles ${ }^{60}$. Esta concepción se opone a la justificación de la legitimidad o el derecho de cada individuo sobre su vida como aspecto central de la ética thoreauviana. Si aceptamos la interpretación que nos ofrece Botkin, resulta que reducimos cualquier valoración de la vida a un antropocentrismo: lo importante es cómo repercute el mundo en lo humano, y no por sí mismo.

Esta postura parece generar muchos problemas en relación a una justificación fuerte de la ética medioambiental thoreauviana. Si lo que está en el punto de mira es conservar la vida o, en sentido más estricto, permitir que la vida se conserve por sí misma en la wilderness, entonces conviene articular nuestros principios de tal modo que el criterio más básico sea el vital y no el racional, espiritual o religioso. Ahora bien, Botkin está considerando que el motivo de que se deba conservar la vida o la wilderness reside en sus repercusiones espirituales y racionales, en que experimentarla es el único modo que tenemos para alcanzar ideas superiores. Pero pensemos del siguiente modo:

Comencemos aceptando la premisa de Botkin, según la cual (1) la especie humana se diferencia del resto principalmente por su capacidad racional, espiritual y religiosa, que le permite alcanzar ideas superiores, que están en un ámbito separado y autónomo de lo meramente material. Sin embargo, siguiendo todavía a Botkin, (2) sólo se puede conseguir el desarrollo de tal capacidad mediante la experiencia de la wilderness. Como consecuencia, (3) los humanos deben conservar la wilderness para poder desarrollar sus capacidades y, para ello, reflexionan sobre cómo actuar conforme a la naturaleza, conforme a la vida en general (lo que para Thoreau supone actuar salvajemente).

(4) Este recurso a la vida iguala a la especie humana con el resto de individuos, ya que tienen en común la actividad de vivir. Por tanto, la salvajez hace que se diluya la distinción categórica antropocéntrica hecha a partir de la capacidad racional humana. Entonces, ¿qué supone, desde el punto de vista de la salvajez, tal racionalidad? Siguiendo a Thoreau: (5) «Es una

\footnotetext{
${ }^{59}$ D. Botkin, No Man's Garden, Island Press, Washington D.C. 2001, p. 38.

${ }^{60}$ Ibid., p. 50.
} 
costra que se caerá suficientemente rápido» ${ }^{61}$, es un medio para conocer el mundo (y en particular la wilderness), pero que sólo suplanta a la sensibilidad para proporcionar cierta estabilidad en forma de ideas y creencias. Tales ideas y creencias carecerían de sentido si no se conservara la wilderness, y en consecuencia desaparecerían (algo imposible si suponemos, como hace Botkin, un dualismo platónico).

El de Concord también afirma que (6) «en la Salvajez está la conservación del mundo ${ }^{62}$. Si mantenemos la postura de Botkin, nos veríamos obligados a considerar que para conservar la wilderness hace falta llegar espiritualmente a la salvajez, como algo superior. (7) Pero como la salvajez nos iguala al resto de la existencia y rompe el antropocentrismo, recurrir a tal espiritualidad nos obliga a considerarla sólo como herramienta en favor de aquello más alto a lo que nos conduce: la conservación de la vida en su sentido más mundano, en la wilderness.

En conclusión, (8) incluso si consideramos un antropocentrismo dualista estricto, la exigencia thoreauviana de salvajez nos abstrae de él y sugiere una concepción de la humanidad como una parte más de la existencia, cuyas capacidades cognitivas, espirituales y religiosas le permiten ser consciente de sus actos, reflexionar sobre ellos y responsabilizarse de lo que hace. Esto le concede a Botkin un punto: la separación entre lo material y lo espiritual. Pero tal separación no implica un platonismo ni un antropocentrismo estricto: sólo señala una separación dentro de lo característico de la especie humana y de su interacción en el mundo. El fundamento para lo espiritual, por lo tanto, no es lo espiritual mismo sino lo vital y salvaje en toda su extensión. Por lo tanto, nos encontramos ante una ética biocéntrica y no antropocéntrica.

Es cierto, tal como indica Botkin, que Thoreau defiende en gran medida la importancia espiritual y racional de las selvas para los humanos; esta importancia se extiende, también, a la vida en general, ya que concibe que la civilización no podría desarrollarse sin alguna zona virgen o salvaje de la que nutrirse (tanto un bosque como un río, un lago, un mar, etc.). Éste podría ser un argumento más que suficiente para su conservación, pero no por ello podemos decir que la ética thoreauviana sea biocéntrica y no antropocéntrica. El motivo fundamental por el que Thoreau considera el beneficio de la wilderness sobre la vida humana es su naturaleza, que no puede ser superada, como vimos en "Paraíso (para ser) recuperado", por una modi-

${ }^{61}$ H.D. Thoreau, The Writings of Henry David Thoreau: Journal, op. cit., p. 105.

${ }^{62}$ H.D. Thoreau, "Walking”, op. cit., p. 224. 
ficación estrictamente racional. Si leemos la ética thoreauviana desde esta perspectiva, la interpretación de Botkin presenta muchas complicaciones, y la única respuesta coherente resulta el biocentrismo.

\section{b. Actualidad de la ética medioambiental thoreauviana}

Para considerar la ética medioambiental de Thoreau en relación con las propuestas contemporáneas, hemos de tener presente este carácter biocéntrico. De tal modo, entre las teorías éticas que podemos considerar al respecto, se encuentra una fundamental: la ecología profunda. Si siguiéramos estrictamente la interpretación que realiza Botkin, esta propuesta constituiría una oposición directa al pensamiento thoreauviano, tal como él mismo indica ${ }^{63}$. Sin embargo, al mostrar que se contradice gravemente con las ideas que hemos expuesto a partir de los textos de Thoreau, cabe considerar una posible concordancia con algunas concepciones de la ecología profunda, expuesta por Arne Naess en siete principios ${ }^{64}$. Entre éstos, hay ciertas diferencias que pueden justificarse con la diferencia temporal y contextual, especialmente política. En cuanto al resto, podemos señalar estos aspectos:

1. Rechazo y sustitución de la imagen del hombre-en-el-entorno en favor de la imagen del campo-total o relacional ${ }^{65}$

La concepción de la vida y la wilderness como ambiente o entorno en el que están incluidos los humanos nos aproxima a esta idea de 'campo-total' o 'campo relacional', que Naess identifica con una interdependencia de todo lo existente. Esta concordancia queda de manifiesto a lo largo de Walden, donde en repetidas ocasiones se propone al individuo humano como parte integrante de la naturaleza ${ }^{66}$.

2. Igualitarismo biosférico ${ }^{67}$

Se trata de un 'criterio general' o amplio que no carece de excepciones, ya que la vida incluye diferencias como que un individuo se coma a otro $^{68}$. Del mismo modo Thoreau acepta que la muerte forma parte de la vida, y no rechaza la caza ni otros tipos de violencia que tienen una justificación vital.

${ }^{63}$ D. Botkin, op. cit., pp. 50, 134.

${ }^{64}$ A. Naess, "The Shallow and the Deep, Long-Range Ecology Movement. A Summary", Inquiry 16 (1973), 95-100.

${ }^{65}$ Ibid., p. 95.

${ }^{66}$ Este tipo de relación con el entorno se manifiesta especialmente en el capítulo "Soledad" de Walden.

${ }^{67}$ Ibid.

${ }^{68}$ Ibid. 
3. Lucha contra la contaminación y el agotamiento de los recursos ${ }^{69}$

Aquí nos encontramos con un problema propiamente contemporáneo. Thoreau no podía pensar directamente en esta cuestión a la hora de plantear su ética, por lo que las pocas referencias que podamos vincular están totalmente descontextualizadas. Eso no significa que se oponga este principio. De hecho, tanto el problema de la contaminación como el de la escasez de recursos son principales para la conservación de la wilderness, y están presentes tangencialmente en la sencillez y la sobriedad thoreauvianas.

4. Complejidad, no complicación ${ }^{70}$

Naess señala que los sistemas naturales son complejos y vastos, son intrincados ${ }^{71}$. El segundo elemento de este principio, que se opone a la complicación, incide sobre que debemos adoptar una actitud adecuada hacia tal complejidad, aceptándola en vez de intentar simplificarla. Thoreau, pese a no ser tan explícito, se refiere a ello con la metáfora de la costra y su rechazo a la domesticación. También conviene tener en cuenta que la simplificación que rechaza Naess no es la sencillez que expone Thoreau, que se refiere a las decisiones que tomamos y no a nuestras concepciones de la naturaleza. Esta oposición a la simplificación tiene que ver, en un sentido más estricto, con la autenticidad frente a lo complejo e inabarcable.

Si aceptamos la importancia contemporánea de las reflexiones de Thoreau respecto al trato con el entorno natural, estas concordancias han de ser nuestro primer punto de apoyo. El hecho de que aceptemos éstas y no otras implica un compromiso específico que va más allá de la intención de conservar el medioambiente: acepta y critica ciertos métodos, que implican cambios en el grado de intervención humana sobre el entorno natural. Si partimos de la interpretación de Cafaro, la vinculación con la ecología profunda y sus propuestas específicas es clara y satisfactoria; sin embargo, hemos de tener en cuenta que aún se conserva, en cierto ideario, la lectura que realiza Botkin, pese a las claras deficiencias que presenta.

\section{Conclusiones}

Tal como se proponía al principio del artículo, hemos hecho un recorrido desde la ética thoreauviana a las posturas actuales sobre el medioambiente. Sobre lo expuesto podemos concluir lo siguiente:

\footnotetext{
${ }^{69}$ Ibid., p. 97.

${ }^{70}$ Ibid.

${ }^{71}$ Ibid.
} 
1. Thoreau elabora una ética sobre el entorno natural biocéntrica, que pone en discusión la naturaleza y las construcciones sociales, exigiendo que se revisen continuamente sus relaciones.

2. La ética medioambiental thoreauviana no tiene un programa teórico determinado, sino que busca la reflexión a partir de la experiencia en el entorno. Ya que tenemos un conocimiento limitado del mundo, nuestra ética nunca puede ser infalible.

3. El biocentrismo thoreauviano tiene en cuenta el factor humano y la racionalidad como aspectos importantes, pero no acepta el antropocentrismo. En contra de algunas interpretaciones, como la de Daniel Botkin, hemos de concluir que Thoreau no centra la importancia de la naturaleza en la vida humana, sino en la vida en general.

4. La propuesta de Thoreau guarda importantes similitudes con la ecología profunda, que pueden observarse en la concepción del mundo como un lugar salvaje y complejo, que se renueva a sí mismo sin la intervención humana, y donde reina el igualitarismo.

5. Para comprender cómo puede ayudarnos el pensamiento de Thoreau a avanzar en la resolución de los problemas medioambientales contemporáneos se hace necesario entender cómo otras posturas actuales han adoptado ideas similares.

\section{Bibliografía}

Botkin, D., No Man's Garden: Thoreau and a New Vision for Civilization and Nature, Washington, D.C., Island Press, 2001.

Burbick, J., Thoreau's Alternative History: Changing Perspectives on Nature, Culture, and Language, Philadelphia, University of Pennsylvania Press, 1987.

https://doi.org/10.9783/9781512819779

Cafaro, P., "Thoreau, Leopold, and Carson: Toward an Environmental Virtue Ethics", Environmental Ethics, 23:1 (2001), 3-17. https://doi.org/10.5840/enviroethics200123135

Cafaro, P., Thoreau's Living Ethics, Athens, University of Georgia Press, 2004.

Casado da Rocha, A., Una casa en Walden, Logroño, Pepitas ed., 2017.

Dobson, A., Pensamiento político verde, Barcelona, Paidós, 1997, trad. José Pedro Tosaus.

Emerson, R.W., Nature, Boston, Thurston, Torry\& Co., 1849. 
Laercio, D., Vidas de los filósofos más ilustres (Libros VIII - X), Buenos Aires, Espasa-Calpe, 1951.

Naess, A., "The Shallow and the Deep, Long-Range Ecology Movement. A Summary", Inquiry 16 (1973), 95-100.

https://doi.org/10.1080/00201747308601695

https://doi.org/10.1080/00201747308601682

Thoreau, H.D., "Walden", en The Writings of Henry David Thoreau, vol. II, Boston, Houghton Mifflin \& Co., 1906, pp. 3-367.

Thoreau, H.D., "Paradise (to be) Regained", en The Writings of Henry David Thoreau, vol. IV, Boston, Houghton Mifflin \& Co., 1906, pp. 280-305.

Thoreau, H.D., "Walking”, The Writings of Henry David Thoreau, vol. V, Boston, Houghton Mifflin \& Co., 1906, pp. 205-248.

Thoreau, H.D., The Writings of Henry David Thoreau: Journal, vol.III, Boston, Houghton Mifflin\& Co., 1906. 